\title{
PREDICTING DRIVER SAFETY IN PARKINSON'S DISEASE: AN INTERIM REPORT OF AN ONGOING LONGITUDINAL STUDY
}

\author{
Ergun Y. Uc, ${ }^{1,2}$ Matthew Rizzo, ${ }^{1,3,4}$ Steven W. Anderson, ${ }^{1}$ JonDavid Sparks, ${ }^{5}$ \\ Qian Shi, ${ }^{5}$ Robert L. Rodnitzky, ${ }^{1}$ Jeffrey D. Dawson, ${ }^{4}$ \\ ${ }^{1}$ Department of Neurology \\ Carver College of Medicine \\ ${ }^{2}$ Neurology Service \\ Veterans Affairs Medical Center, \\ ${ }^{3}$ Department of Mechanical and Industrial Engineering \\ ${ }^{4}$ Public Policy Center, \\ ${ }^{5}$ Department of Biostatistics \\ College of Public Health \\ University of Iowa, Iowa City, USA \\ E-mail: ergun-uc@uoiwa.edu
}

\begin{abstract}
Summary: This article summarizes the baseline results of an ongoing longitudinal, NIH-funded study on prediction of driver safety in patients with Parkinson's disease (PD). Patients with even mild to moderate PD who drive and live independently suffer from visual and cognitive dysfunction, which appear to be the main contributors to decreased driving performance and safety, rather than the motor dysfunction for which PD is known.
\end{abstract}

\section{OBJECTIVES}

To understand the mechanisms of driver safety errors in Parkinson's disease (PD) and to develop a reliable and efficient battery of tests to identify PD drivers at risk for crashes.

\section{Background}

PD is a relatively common neurodegenerative disorder that affects about one million Americans. PD impairs motor function (e.g., tremors, rigidity), cognition, vision, mood, and sleep, and may increase risk for crashes. However, the severity and course of these impairments vary widely among PD patients, and there are no established guidelines on how to advise drivers with PD on driving safety.

\section{METHODS}

Longitudinal project to study the driving abilities and crash risk in PD using annual testing of the cognitive/visual/motor functions (the "off-road battery"), experimental drives in an instrumented vehicle (IV) and a high-fidelity driving simulator (SIM), and state DOT crash and citation records. 


\section{RESULTS}

Results from baseline (year 1) evaluation comparing licensed, active drivers with PD (DPD) and elderly control drivers (CD) with no neurological disease:

1) Off-road battery (Uc et al., 2005): Although none of the DPD ( $\mathrm{n}=76)$ met dementia criteria and most had only mild-moderate disease severity, they performed significantly worse than $\mathrm{CD}(\mathrm{n}=161)$ on all motor, cognitive, and visual tests $(\mathrm{p}<0.001$ to $\mathrm{p}<0.05)$, including Useful Field of View (UFOV). Cognitive and visual impairments correlated with worse scores on axial motor functions and contributed to disability.

2) Driving Habits Questionnaire (Uc et al., 2007a): Although there were no significant group differences in self-reported crash and citation rates and driving exposure, $26 \%$ of DPD $(n=97)$ rated their driving ability unfavorably compared to $8 \%$ of $C D(p<0.001)$. Compared to $\mathrm{CD}$, larger proportions of DPD reported driving slower than the normal traffic flow, avoiding driving in rain, at night, or on high-volume roads, or merging and making lane changes due to difficulties, all $\mathrm{p}<0.05$.

\section{IV experiments:}

1) Visual search (Uc et al., 2006a): DPD $(n=79)$ identified fewer landmarks and traffic signs compared to $\mathrm{CD}(\mathrm{n}=151)$ along a 1-mile, 4-lane commercial strip $(\mathrm{p}<0.001)$, which was predicted by poorer performance on UFOV and visuospatial abilities. DPD committed more at-fault safety errors during the task than $\mathrm{CD}$ even after adjusting for baseline errors $(\mathrm{p}<0.001)$, which was predicted by decreased cognitive flexibility.

2) Audio-verbal distraction (Uc et al., 2006b): Paced Auditory Serial Addition Task (PASAT) was administered to 71 DPD and 147 CD while they drove on a 4-lane interstate freeway. Compared to baseline segment, the at-fault error count increased in $28.2 \%$ of DPD vs. $15.8 \%$ in $\mathrm{CD}, \mathrm{OR}(95 \% \mathrm{CI})=2.6(1.2-5.7), \mathrm{p}=0.016$. Decreased performance on tests of cognitive flexibility, verbal memory, postural control, and increased daytime sleepiness predicted worsening of driving performance due to distraction within DPD.

3) Route following (Uc et al., 2004): Compared to CD ( $n=162)$, DPD ( $n=77)$ made more incorrect turns, got lost more often, and committed more at-fault safety errors $(p<0.001)$, which were predicted by UFOV and visuospatial memory impairments.

\section{SIM experiments:}

1) Motor distraction (Uc et al., 2006c): Window Rolling Task (WRT) was administered to 31 DPD and 19 CD while they drove on a 2-lane rural highway. DPD took more time to complete the WRT compared to controls (21.5 vs. $13.5 \mathrm{sec}$, medians) and had a higher standard deviation of lane position during the WRT, all $\mathrm{p}<0.01$. Compared to baseline segment, the at-fault error count increased in $58.6 \%$ of DPD vs. $15.6 \%$ in CD, OR $(95 \%$ $\mathrm{CI})=7.6(1.8-31.8), \mathrm{p}<0.001$, which was predicted by impaired visuospatial abilities and low activities of daily living scores.

2) Intersection incursion in low-contrast lighting conditions (Uc et al., 2007b): Sixty-seven DPD and $51 \mathrm{CD}$ drove on a 2-lane rural highway under foggy conditions in a scenario in which the driver's approach to within 4.0 seconds of an intersection triggered an illegal incursion by another vehicle causing crash risk. $76.1 \%$ of DPD crashed compared to 
$37.3 \%$ of $\mathrm{CD}, \mathrm{OR}(95 \% \mathrm{CI})=5.4(2.4-11.9), \mathrm{p}<0.0001$. The time to first reaction (releasing accelerator, braking, or steering away) of drivers with PD was slower than that of controls (median 2.7 vs. 2.1 seconds, $\mathrm{p}<0.0001$ ), which correlated with poor performances on tests of visual perception and memory, visuospatial abilities, executive functions, and motor function (all $\mathrm{p}<0.05$ ). Prolonged reaction time and decreased performance on tests of contrast sensitivity, perception of structure from motion, UFOV, and visuospatial perception predicted crashes (all $\mathrm{p}<0.05)$.

\section{CONCLUSIONS}

Drivers with PD in our study appear to have insight into their driving difficulties and report compensatory self-restricting of risky driving circumstances, which may explain the lack of differences in self-reported crashes or moving violations compared to controls at baseline. This suggests that a diagnosis of PD alone is not sufficient to trigger a license restriction or revocation. However, favorable self-reports of DPD do not prove that they can drive as safely as CD. When DPD are subjected to standardized, rigorous driving tests in the IV and SIM, they show decreased driving performance and increased safety errors compared to CD.

Impairments in cognition, vision, mood, and sleep are present even in the early stages of PD, and have more effect on driving ability than the typical motor dysfunction. Thus, a good testing battery for accurate and fair prediction of driver safety in PD should assess both the well-known motor dysfunction and the lesser known but widely present non-motor aspects of the disease such as cognitive, visual, and sleep dysfunction.

\section{ACKNOWLEDGMENTS}

This study was supported primarily by the NIH/National Institute of Neurological Disorders and Stroke grant R01 NS044930 (Predicting Driver Safety in Parkinson's Disease) to EYU; as well as by the National Institute on Aging grants R01 AG 17717 and R01 AG 15071 to MR, and the University of Iowa-Carver College of Medicine/College of Public Health New Investigator Research Award to EYU.

\section{REFERENCES}

Uc, E.Y., Rizzo, M., Anderson, S.W., Shi, Q., Dawson, J. (2004). Driver Route-Following and Safety Errors in Parkinson's Disease. Presented at the American Academy of Neurology $56^{\text {th }}$ Annual Meeting, April 25-May 1, San Francisco, CA. Neurology, 62(7) Supplement 5: A523.

Uc, E.Y., Rizzo, M., Shi, Q., Anderson, S.W., Rodnitzky, R.L., Dawson, J. (2005). Visual Dysfunction in Parkinson's Disease without Dementia. Neurology, 65(12): 1907-13.

Uc, E.Y., Rizzo, M., Anderson, S.W., Sparks, J., Rodnitzky, R.L., Dawson, J.D. (2006a). Impaired Visual Search in Drivers with Parkinson's Disease, Annals of Neurology, 60: 407413.

Uc, E.Y., Rizzo, M., Anderson, S.W., Sparks, J., Rodnitzky, R.L., Dawson, J.D. (2006b). Driving with Distraction in Parkinson's Disease. Neurology, 67: 1774-80. 
Uc, E.Y., Rizzo, M., Anderson, S.W., Sparks, J., Rodnitzky, R.L., Dawson, J.D. (2006c). Effect of Concomitant Motor Task on Driving in Parkinson Disease. $10^{\text {th }}$ International Congress of Parkinson's Disease and Movement Disorders, Kyoto, Japan, October 28-November 2. Movement Disorders, 19, Supplement 9: S193-4

Uc, E.Y., Rizzo, M., Anderson, S.W., Sparks, J., Rodnitzky, R.L., Dawson, J.D. (2007a). An analysis of Driving Habits in Parkinson's Disease. Presented at the Transportation Research Board $86^{\text {th }}$ Annual Meeting, January 20-24, Washington, DC. Proceedings CD-ROM.

Uc, E.Y., Rizzo, M., Anderson, S.W., Sparks, J., Rodnitzky, R.L., Dawson, J.D. (2007b). Increased Crash Risk in Drivers with Parkinson s Disease under Low Visual Contrast Lighting Condition. Presented at the American Academy of Neurology $59^{\text {th }}$ Annual Meeting, April 28-May 5, Boston, MA. Neurology, 68(12) Supplement 1: A208. 